\title{
Analysis of Utility Theory on VLSI Cell Placement
}

\author{
R. Manikandan*, P. Swaminathan and V. Vaithiyanathan \\ School of Computing, SASTRA University, Thanjavur, 613401, India
}

Received: 16 Jul. 2013, Revised: 18 Oct. 2013, Accepted: 19 Oct. 2013

Published online: 1 Jul. 2014

\begin{abstract}
This paper focuses on the employment of utility theory for decision making under risk and uncertainty. Further, we have also investigated the efficiency of the three types of utility curves namely Conservation Man, Average player and the Gambler. The utility theory has been analyzed for its efficiency to solve cell placement problem.
\end{abstract}

Keywords: Utility theory, VLSI cell placement, Maximizing utility, Decision utility, Common ratio effect, Legrangian Multiplier

\section{Introduction}

General utility refers to the way people assign value based on their ordering of preferences $[1,2,3,4,5]$. Utility is a measure of the satisfaction a person derives from choosing a good, a course of action, or a lottery that offers chances of altering a good. Utility theory methods take advantage of individuals subjective risk perception to derive values for objects or decisions. Most phases of VLSI design automation comprise very large and complex combinatorial optimization problems with numerous constraints and very noisy solution space. Physical design of VLSI Circuits constitutes the conversion of structural representation into layout representation of circuits. Structural representations describe the system in terms of logic components and its interconnects. Layout representations define circuits in terms of a set of geometric objects which specify the dimensions and locations of transistors and wires on a silicon surface. One of the most critical problems encountered in the design of VLSI Circuits is assigning locations to circuit modules and routing the connections among them such that the ensuing area is minimized. The complexity of the problem has mandated the partitioning the VLSI Circuits into two consecutive stages. The first stage, commonly referred to as the placement problem, deals with assigning locations to individual modules. The quality of the routing obtained at the second stage depends critically on the placement output of the first stage. Hence the goal of a good placement techniques is to position the cells such that the ensuing area is minimized, while the wire lengths are subject to critical length constraints. Walker[6] provides defense for unified notion of utility that does not need risk for its existence, but that has relevance for risk. Walker observes that the development of expected utility theory by Von Neumann and Morgenstern [5] was motivated by their desire to obtain a cardinal utility that is relevant to the game theory. In the past, the principal of the VLSI placement has been minimization of interconnect wire-length. In standard cell layout style all the circuits modules or cell are constrained to have the same height, while width of the cell is variable and depends upon its complexity [7]. Cells are placed in horizontal rows and the cell rows are separated by horizontal routing channels. Module placement is an NP-hard problem and therefore, cannot be solved exactly in polynomial time. Trying to get an exact solution by evaluating every possible placement to determine the best one would take time proportional to the factorial of the number of modules. The utility framework requires the outcome probabilities to obtain proposed well configurations. In some cases the determination of outcome probabilities might be computationally infeasible, particularly for very large numerical modules.

A second approach is presented in which the well placement problem has been formulated as the optimization of a random function which does not require the prior knowledge of outcome probabilities. Hence, in this paper, the notion of utility theory is focused in both decision making under risk and uncertainty. Also we have investigated the efficiency of selection of three types of utility curves, such as Conservation Man, Average player and the Gambler. Further, the utility theory has been

\footnotetext{
*Corresponding author e-mail: manikandan75@core.sastra.edu
} 


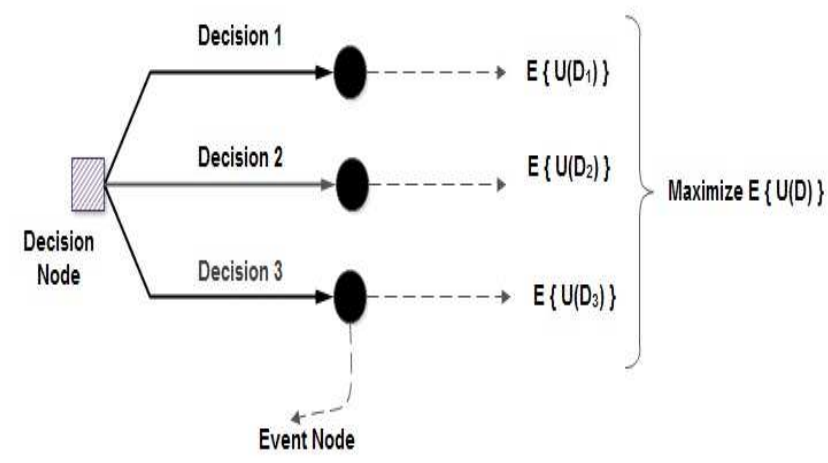

Fig. 1: The well placement decision tree with event nodes

analyzed for its efficiency to solve cell placement problem.

\section{Utility Theory and Utility Functions}

We define decision under certainty as "the decisions taken under certainty of action results invariably in a specific outcome (prospect, alternative, etc)" We propose that a decision is taken under risk if each action of the decision results in a set of possible specific outcomes, with each outcome occurring within a known probability. Utility theory and Utility functions are applicable to decision making under uncertainty or under risk. The whole process of decision tree construction and definition of the problem as the maximization of expected utility rather than the monetary value constitutes a transformation of the problem according to the decision makers attitude towards risk utility or preference theory, which explains how this transformation is made possible [8]. The utility function is a tool which to quantifies the decision makers risk attitude [9].

Based on the shape of the utility function, the nature of the decision maker can be determined and classified as either, risk prone, risk averse or risk neutral (Fig. 1). A decision maker, who is risk neutral, has a linear utility function which is equivalent to basing decisions purely on monetary value (Net present value(NPV)). A risk-averse decision maker has a concave utility function which corresponds to the avoidance of uncertain areas of search space event if they might have the possibility of greater financial gain. The decision maker, who is risk prone, is willing to take some risk for greater financial gain [3], hence has a convex utility function. The exponential form of a simple analytical utility function is represented as

$$
U(x)=a+b^{-r x}
$$

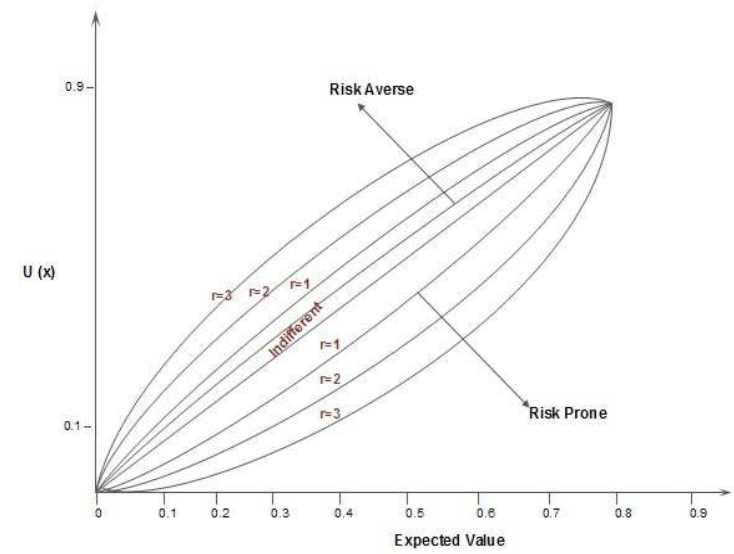

Fig. 2: The exponential utility function for different exponent values, $\mathrm{R}$

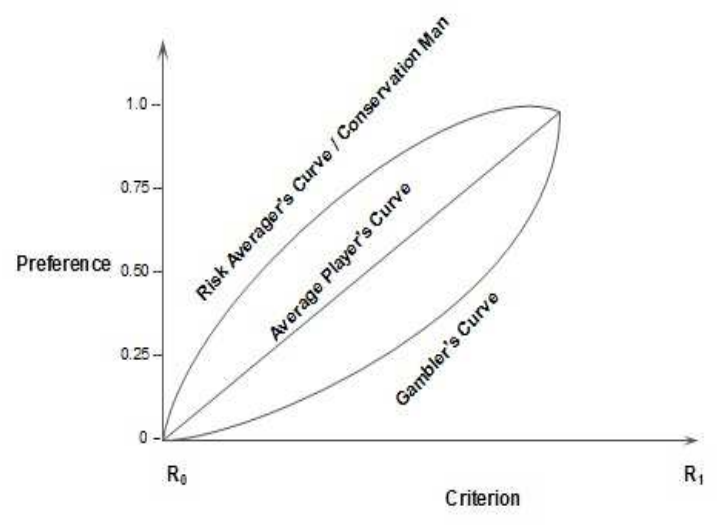

Fig. 3: Utility Curve

Where ' $\mathrm{x}$ ' is the objective function value which is NPV in this case. A normalized version of (1), with $a=1$ and $b=-1$ is visualized in Fig.2. The curvature of the utility function determines the risk attitude of the decision maker as $\phi$ in Fig.2. The magnitude of risk aversion of a given utility function $U$, is given by

$$
R(x)=-\frac{U^{\prime \prime}(x)}{U^{\prime}(x)}
$$

The term $\mathrm{R}(\mathrm{x})$ in (2) is also referred to as the ArrowPratt measure of absolute risk aversion or the risk aversion coefficient[8]. The risk aversion coefficient is a constant for exponential utility function and is equal to the exponent $r$ in equation (2).

There are three different types of curve, which correspond to three different attitudes toward risk.

1. The Conservation Man: The conservation mans curve is concave in shape, which is typically observed in 
practice. The conservation man is averse to risk.

2. The Average Player: This persons curve is a straight line, which means that he/she wishes to play the long-run averages.

3. The Gambler: This person is the rarest of the three types, his utility curve is convex in shape and is prone to take more risk to gain financially.

\section{Axioms of Utility Theory}

We define decision under certainty as "the decisions taken under certainty of action results invariably in a specific outcome (prospect, alternative, etc)" We propose that a decision is taken under risk if each action of the decision results in a set of possible specific outcomes, with each outcome occurring within a known probability. Utility theory and Utility functions are applicable to decision making under uncertainty or under risk. The whole process of decision tree construction and definition of the problem as the maximization of expected utility rather than the monetary value constitutes a transformation of the problem according to the decision makers attitude towards risk utility or preference theory, which explains how this transformation is made possible [8]. The utility function is a tool which to quantifies the decision makers risk attitude [9].

\section{Analysis of Utility Curve}

Analysis of Utility Curve involves the following steps:

1. Defining the alternative courses of action.

2. Defining a risk-taking attitude in the form of utility curve.

3. Choosing a course of action that is optimal, given the risks and risk-taking attitude of the indivudual.

4. Using that curve to convert economic consequences into utilities.

5. Selecting that course of action that has the highest expected utility.

\section{Rules for Maximizing utility}

Suppose that a customers preference can be represented by the utility function, $\mathrm{U}=\mathrm{f}(\mathrm{X}, \mathrm{Y})$ where $\mathrm{X}$ and $\mathrm{Y}$ represent the amount of goods $\mathrm{X}$ and $\mathrm{Y}$ used. The objective of the consumer is to ensure that the utility is maximized within the constraint that the total expense do not supersede the income (I).

$$
I=P_{X}^{X}+P_{Y}^{Y}
$$

To solve this problem, we form the function $V=F(X . Y)+\lambda\left(1-P_{X}^{X}-P_{Y}^{Y}\right)$. (This function is called a "legrangian" and the variable $\lambda$ is known as the
"Legrange multiplier"). In this function, if the values of $X$ and $Y$ satisfy the constraint, $I-P_{X}(X)-P_{Y}(Y)=0$ and $\lambda$ is not equal to zero, then the values of $\mathrm{V}$ and $\mathrm{U}$ are the same. Maximizing $\mathrm{V}$ is equivalent to maximizing $\mathrm{U}$ subject to the income constraint. To maximize utility, all the partial derivatives of this legrange equation must be set to zero.

$$
\begin{gathered}
\frac{\partial V}{\partial X}=\frac{\partial F}{\partial X}-\lambda P X=0 \\
\frac{\partial V}{\partial Y}=\frac{\partial F}{\partial Y}-\lambda P Y=0 \\
\frac{\partial V}{\partial \lambda}=I-P_{X} X-P_{Y} Y=0
\end{gathered}
$$

Note that this condition fulfills the requirement imposed by the budget constraint. Assuming that the second-order conditions for a maximum are fulfilled, equations (4-5) can be solved for the utility. Maximizing values of $\mathrm{X}, \mathrm{Y}$ and $\lambda$, the first equation can be solved for $\lambda$ to obtain $\lambda=\frac{\partial F / \partial X}{P_{X}}$ and from the second equation, $\lambda=\frac{\partial F / \partial Y}{P_{X}}$ can be derived. Since both these expression are equal to $\lambda$, therefore, they should also be equal to each other.

$$
\lambda=\frac{\partial F / \partial X}{P_{X}} \quad \lambda=\frac{\partial F / \partial Y}{P_{X}}
$$

The terms in the numerators are the marginal utilities of goods $\mathrm{X}$ and $\mathrm{Y}$, which is the expected condition. Maximum utility is achieved when the marginal utility per dollar of each good is the same.

$$
\frac{M U_{X}}{P_{X}}=\frac{M U_{Y}}{P_{Y}}
$$

Consequently, the equation (6) implies that the consumers budget must have been exhausted as well. Results for effectiveness of implementation of VLSI cell placement are presented. The results demonstrate the placement of a set of cells on the VLSI layout. The netlist in the VLSI layout are interconnected with each of the cell and with the library having the layout information for each type of cell. Our consumers VLSI circuit design problem under utility function $\mathrm{U}=\mathrm{f}(\mathrm{X}, \mathrm{Y})$ is to maximize utilization in each VLSI Cell placement subject to the constraints and the total expenditures on $\mathrm{X}$ and $\mathrm{Y}$ are equal. Hence the demonstrated online VLSI cell placement technique aims at equalizing the utility value across all applications while also satisfying restrictions pertaining to the operations, such that over allocation of memory is prevented and the number of placement changes minimized. Among all utility curves, the conservation man of Maximal utilities of $\mathrm{X}$ an $\mathrm{Y}$ which gives the averages risk by legrangian multiplier and the total expenditures on $\mathrm{X}$ and $\mathrm{Y}$ are equal.

Example: Given the consumers income, $\mathrm{M}$ and prices, $P_{X}$ 


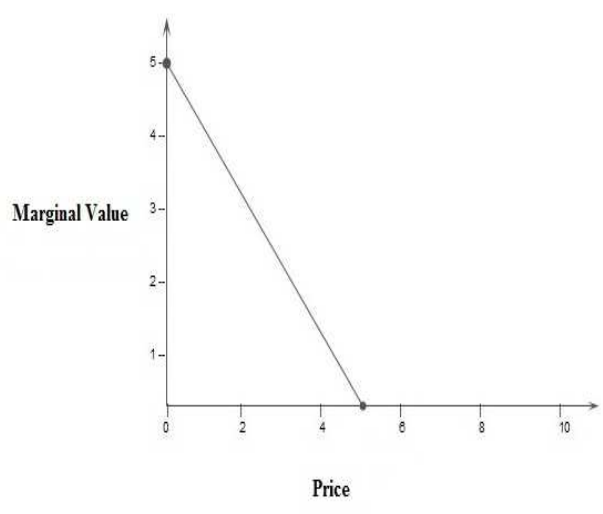

Fig. 4: Maximizing utility

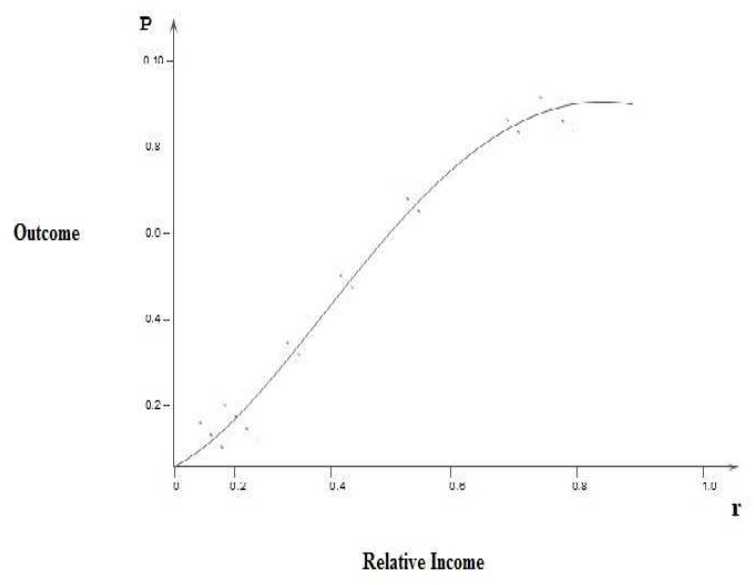

Fig. 5: Decision Utility $p=D(r)$ and $P_{Y}$, the consumers problem is to choose the affordable bundle that maximizes his/her utility. The feasible set (budget set) maintains that the total expenses made should not be more than the income, which is represented as

$$
P_{X} X+P_{Y} Y \leq M
$$

Since everyone expects more income, equation (9) must be equal and represent as solution to the consumers problem. $U(x, y)=x y, M=10, P_{x}=1, P_{y}=1$ the legrange expression $L=x y+\lambda[10-x-y]$ the first order conditions are

$$
\begin{gathered}
y-\lambda=0 \\
x-\lambda=0 \\
10-x-y=0
\end{gathered}
$$

First solve the first two equations (10) and (11) for $\lambda$ and set the expressions equal to each other. $\lambda=y=x$ now solve the other equation (12) ( the budget constraint) for either $\mathrm{x}$ or $\mathrm{y}$ in terms of the other. $\mathrm{y}=10 \mathrm{x}$. Finally, substitute this equation into the earlier legrange equation $10-\mathrm{x}=\mathrm{y}$. By doing so, we get $\mathrm{x}=5$. Substitute the answer for $x$ into the budget constraint to get $y=5$. Thus it is observed that the marginal rate of substitution equals the price ratio at the point $(5,5)$.

\subsection{Decision Utility:}

The utility curves for different lottery ranges appears to have a similar shape. This leads to the hypothesis that the utility may be described within the range. The hypothesis is tested by linearly transforming all the outcomes and certainty equivalents to $[0,1]$ interval using

$$
r=\frac{C_{e}-P_{\min }}{P_{\max }-P_{\min }}
$$

where $\mathrm{r}$ denotes the relative outcome, $C_{e}$ denotes the certainty equivalent, $p_{\max }=\operatorname{Max}(r)$ is the maximum lottery outcome, and $p_{\text {min }}=\operatorname{Min}(x)$ is the minimum lottery outcome. All relative outcomes ' $r$ ' together with their respective probability $\mathrm{p}$ are then presented on a single graph. When the points are plotted very closely, an $\mathrm{S}$-shape curve is obtained, which can be easily estimated. A decision utility function ' $\mathrm{D}$ ' defined as $\mathrm{p}=\mathrm{D}(\mathrm{r})$ can be determined for outcomes expressed in relative terms.

Decision utility is very much different from the kind of utility assumed by Von Neumaan and Mongestern[5], although it has been derived using their original method. It focuses on the description of decisions made under conditions of risk. It does not focus on the way people recognize either income or other welfare levels. Therefore, the decision utility is converted into relative certainty equivalent

$$
C_{e r}=D^{-1}(P)
$$

such that it can be transformed to its absolute value

$$
C_{e}=p_{\min }+C_{e r}\left(P_{\max }-p_{\min }\right)
$$

For $p_{\min }$ equation (15) simplifies to

$$
C_{e}=p_{\max } D^{-1}(P)
$$

This method bases its presumption on intuition that allows for fundamental measurements instead of simple option ordering. 


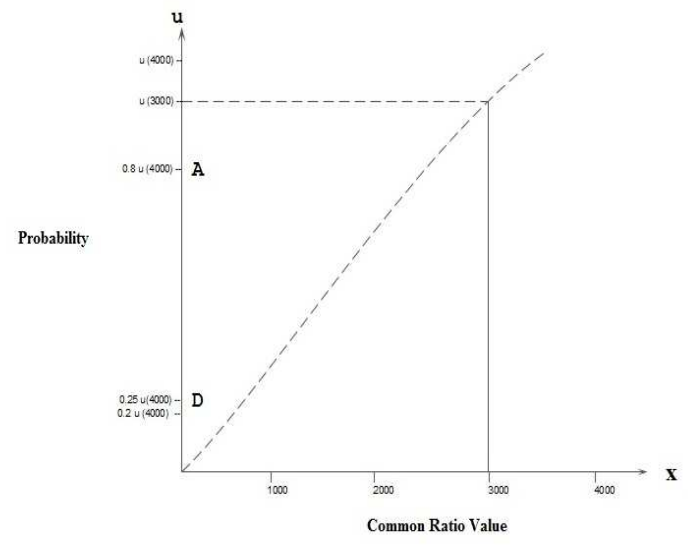

Fig. 6: Expected Utility

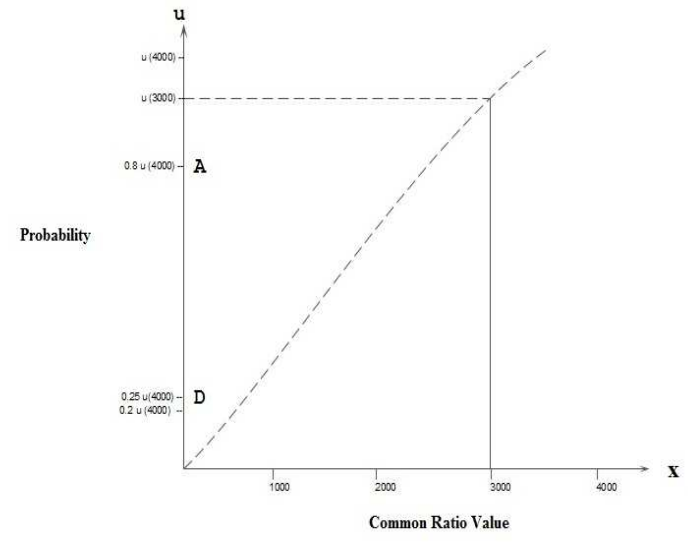

Fig. 7: Decision Utility

\subsection{Utility of Common Ratio effect:}

The Common Ratio effect analyzed by Kahnemann and Tversky [10] can be explained using the decision utility model.

Problem I: Choose between

A: 4000 with a probability of 0.80 or 0 with a probability 10.20

B: 3000 with a probability of 1.00

Problem II: Choose between

C: 4000 with a probability of 0.20 or 0 with a probability of 0.80

D: 3000 with a probability 0.25 with a probability of 0.75

Experimental results consistently reveal that most people choose option B in problem I and option $\mathrm{C}$ in problem II. Expected Utility theory, per contra predicts that people would choose either (A and C) or (B and D), as the probabilities of winning the main prize in the second pair of choices differ by a common ratio factor of 4 compared with the first pair. These options are represented on the utility axis in Figure 6.

The solution of this problem demonstrates how a single decision utility applied to different lottery ranges predict inconsistent choices (Fig.7). The shape of both the utility curves is the same as they are restated decision utility functionaries. The range of the red curve is $[0,4000]$ as this corresponds with options A and C, which has a maximum outcome of 4000 . The range of the blue curve is $[0,3000]$ as this corresponds with options B and $\mathrm{D}$, which has a minimum outcome of 3000 . For greater probabilities, option $\mathrm{B}$ is better than option $\mathrm{A}$. In the case of lower probabilities, option $\mathrm{C}$ is better than $\mathrm{D}$. This model is compared with utility function (curves) based on the channel router concept. The each mark A, B, C, D suggested by them help us to estimate the parameters. We divide the channel routing problem domain in VLSI placement to give more effective and efficient techniques to handle existing difficulties and design complexities. We have to adopt combination of Common Ratio effect Methodologies on the strengthen avoid weakness in single VLSI Cell placement. The above decision utility model to determine the best location of each cell .So as to minimize the total area of the layout and the length of the nets connecting the cells together. Finally in decision utility (Figure 5.7) option B prevails over option A, C is better option than D. The best location of VLSI cell placement by Common Ratio effect is ' $\mathrm{C}$ ' and the length of the nets connecting the cells together.

\section{Conclusion and Future work:}

We analyze the utility properties of utility curves, rules for Maximizing utility curve, Decision utility and Common ration effect. Also we have investigated the efficiency of selection of three types of utility curves, such as Conservation Man, Average player and the Gambler which gives the expected value and Risk average of the decision maker A,B,C and D which occupy in VLSI cell placement. Further, the utility theory has been analyzed for its efficiency to solve cell placement problem. Similar results based on utility idea in VLSI Circuit problems can be obtained using Max-Min approach partitioning and minimize the expected utility.

\section{References}

[1] Luce, R. D., and H. Raiffa. Games and decisions: Introduction and critical survey. NewYork: John Wiley, (1957).

[2] Myerson, R. B., An axiomatic derivation of subjective probability, utility, and evaluation functions. Theory and Decision, 11, 339-52 (1979). 
[3] Raiffa, H., Decision analysis: Introductory lectures on choices under uncertainty . NewYork: Random House, (1968).

[4] Raiffa, H., Decision Analysis, Menlo Park, CA: AddisionWesley, (1968).

[5] Von Neumann, John and Oscar Morgenstern, Theory of Games and Economic Behavior, Princeton, NJ: Princeton University Press, (1944).

[6] Smith, Vernon L. and Walker, James M., Rewards, Experience, and Decision Costs in First Price Auctions. Economic Inquiry, 31, 237-44 (1993).

[7] Sait, S. M., Youssef, H., VLSI Physical Design Automation: Theory and Practice. McGraw-Hill Book Company, Europe (1995).

[8] Holloway, C. A., Decision Making under Uncertainty, Englewood Cliffs: Prentice Hall, (1979).

[9] Rubinstein, M., Security Market Efficiency in an ArrowDebreu Economy, American Economic Review, (1975).

[10] Kahneman, D. and Tversky, A., Prospect theory: an analysis of decision under risk,Econometrica, 47, 763-791 (1979).

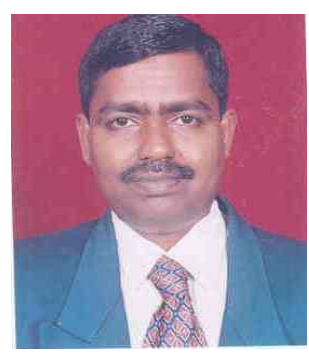

R. Manikandan obtained the Bachelor Degree in Computer Science and Engineering at Bharathidasan University (Tiruchirapalli) in the period of 1992-96 and obtained the Master Degree in VLSI Design at SASTRA University (Thanjavur) in the period of 2001 02. Now, he is currently working as Senior Assistant Professor (SAP) in the Department of Information and Communication Technology at SASTRA University and moreover he is pursuing the Ph.D. degree as Part-Time at the same university. His area of interest includes VLSI Physical Design and Data Mining. He has published more than 20 research papers in the various fields.

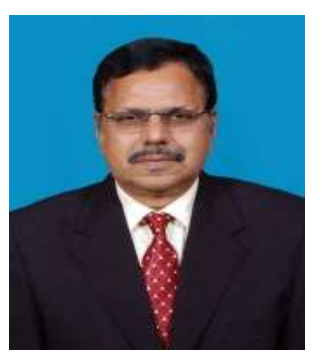

\begin{tabular}{lrr} 
P. & \multicolumn{2}{r}{ Swaminathan } \\
holds & Honours degree \\
in & Electronics & and \\
Communication Engineering \\
and & Doctorate & degree
\end{tabular} in Electronics Engineering. $\mathrm{He}$ is Fellow of Institution of Engineers, India. Currently, he is working as Dean in School of Computing at SASTRA University. His research interest includes Embedded Systems, Software Engineering and Expert Systems.

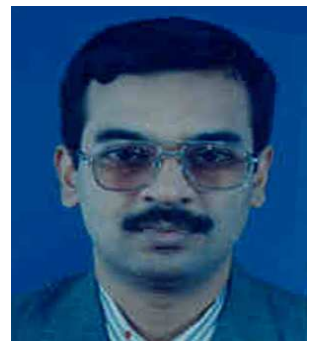

V. Vaithiyanathan

obtained Doctorate

Degree from Alagappa university,India. Currently, he is working as Associate Dean in School of Computing at SASTRA University. His research interest includes Image Processing, Data mining and network security. 